INRA Prod. Anim., 2010, 23 (1), 5-10

\title{
Robustesse, rusticité, flexibilité, plasticité... les nouveaux critères de qualité des animaux et des systèmes d'élevage : définitions systémique et biologique des différents concepts
}

D. SAUVANT, O. MARTIN

INRA, UMR791 Modélisation Systémique Appliquée aux Ruminants, 16 rue Claude Bernard, F-75231 Paris, France AgroParisTech, Modélisation Systémique Appliquée aux Ruminants, 16 rue Claude Bernard, F-75231 Paris, France

Courriel :daniel.sauvant@agroparistech.fr

Les notions de rigidité, d'élasticité, de flexibilité et de plasticité se retrouvent dans de nombreuses disciplines scientifiques, qu'il s'agisse de la physique, de la sociologie, de l'économie, des arts, de la biologie, etc. Dans le langage courant, elles sont également utilisées et y font sens de façon souvent intuitive. Elles permettent au sens large de qualifier la réaction à une contrainte. Cette problématique se pose en sciences animales, qu'il s'agisse de niveaux d'organisation biologiques tels que la cellule, l'organe, l'individu ou de tels que le système de production et la filière. En préambule, il nous semble important de fournir les définitions les plus partagées des différents mots-clés et des concepts sous-jacents du thème niveaux d'organisation biotechniques

de ce dossier de la revue INRA Productions Animales, de manière à pouvoir les appliquer dans la mesure du possible avec des sens proches dans les différents secteurs disciplinaires où ils peuvent être utilisés.

Dans toutes les situations rencontrées, et pour tous les niveaux d'organisation considérés, l'objet d'étude est envisagé comme un système. Un système est défini au minimum par sa frontière et les éléments qui le composent et qui sont liés entre eux, et avec l'extérieur, par des réseaux de flux de matière et $d$ 'information. Compte tenu du fait que la majorité des articles de ce dossier concernent l'organisme animal, celui-ci sera le plus souvent cité dans les exemples.

Figure 1. Réponse dynamique d'un système à une perturbation.

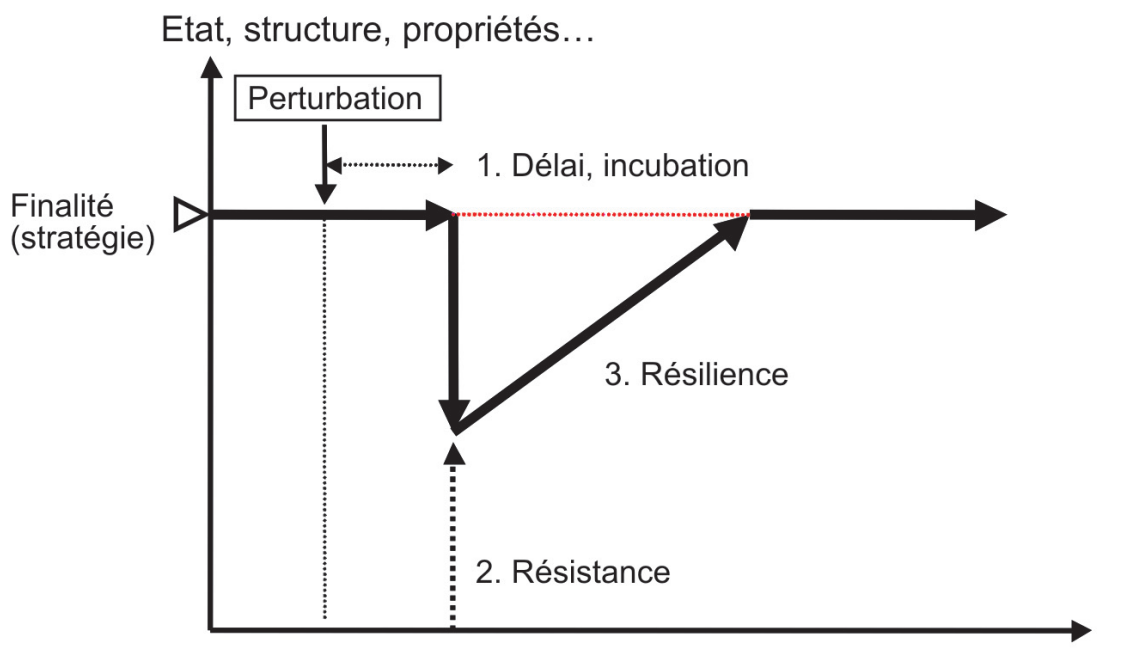

Temps
1 / Les composantes des réponses adaptatives à une perturbation

\section{1 / Situation élémentaire}

En première approche, la problématique concerne les réponses adaptatives d'un système à une perturbation, agissant comme une contrainte vis-à-vis de son fonctionnement. Cette contrainte peut être externe ou interne au système. La figure 1 résume la dynamique de cette réponse qui s'observe au niveau de l'état, c'est-à-dire la structure et/ou la fonctionnalité du système considéré. Au début, le système est sur sa trajectoire d'équilibre. Ensuite, une perturbation plus ou moins soudaine et intense entraîne, après un certain délai, ou phase d'incubation, la réponse du système considéré. L'amplitude de cette réponse dépend de l'intensité de la perturbation et du degré de résistance du système à celle-ci. Cette amplitude de réponse est parfois évaluée relativement à l'intensité de la perturbation, en termes de niveau d'élasticité (cf. les sciences sociales). Dans la phase suivante, le système tend à revenir sur sa trajectoire initiale ou sur une nouvelle trajectoire. Les modalités de ce retour (vitesse de retour, degré de celui-ci...) déterminent la capacité de récupération, ou de régénération du système, appelée résilience dans sa définition la plus fréquente.

La figure 1 présente volontairement un cas trivial de manière à pouvoir présenter les principales composantes des critères de qualité des animaux d'élevage. Dans la réalité, chaque caractère considéré d'un système présente sa pro- 
Figure 2. Modalités des réponses des systèmes à une perturbation.

$$
\text { FINALITE (téléonomie) }
$$

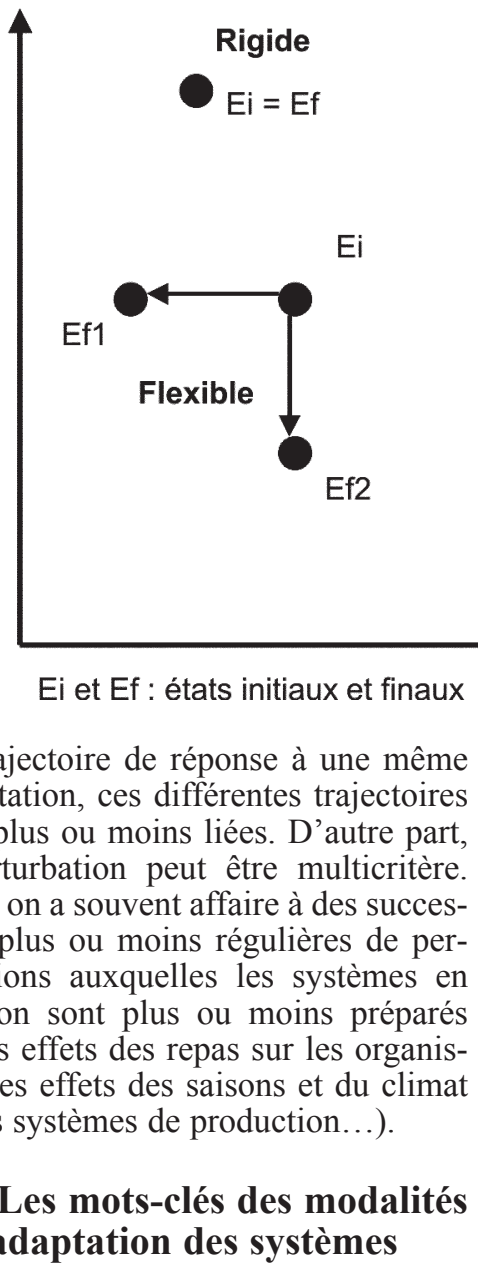

pre trajectoire de réponse à une même sollicitation, ces différentes trajectoires étant plus ou moins liées. D'autre part, la perturbation peut être multicritère. Enfin, on a souvent affaire à des successions plus ou moins régulières de perturbations auxquelles les systèmes en question sont plus ou moins préparés (cf. les effets des repas sur les organismes, les effets des saisons et du climat sur les systèmes de production...).

\section{2 / Les mots-clés des modalités de l'adaptation des systèmes}

La réponse adaptative des systèmes aux perturbations s'exprime selon différentes modalités. En effet, cette adaptation aux perturbations peut consister à :

- résister, on parle alors de systèmes rigides,

- se déformer, on parle alors de systèmes élastiques ou flexibles,

- se transformer, on parle alors de systèmes plastiques.

D'une façon générale, on considère que la capacité d'adaptation confère une certaine stabilité (de structure, de fonctionnalité, de finalité...) face aux changements et aux perturbations. En d'autres termes, ces questions relèvent du degré d'autonomie des systèmes.

L'adaptation comprend plusieurs composantes et on distingue, en particulier, les composantes statiques et dynamiques. Dans le cas des systèmes considérés dans ce dossier, on se place le plus souvent dans un contexte dynamique, l'approche statique étant généralement utilisée pour faciliter un premier stade de la compréhension. ture et/ou sa finalité. re éventuelle.
Les systèmes élastiques s'adaptent en modifiant transitoirement leur structure, leur résilience étant donc égale à leur déformation maximale. En revanche, on considère généralement que leur finalité n'est pas modifiée, on parle alors de stabilité téléonomique. L'homéostase (cf. ci-dessous) traduit les propriétés élastiques des êtres vivants face aux différentes perturbations auxquelles ils sont confrontés. Les adaptations tactiques des systèmes traduisent également souvent des propriétés élastiques.

Les systèmes flexibles s'adaptent aux perturbations grâce à leur capacité à modifier soit leur structure tout en gardant la même finalité, soit leur finalité tout en conservant leur structure. La flexibilité implique généralement l'idée d'une gamme de formes possibles.

Les systèmes plastiques s'adaptent 1.3 / Les définitions des modalités de l'adaptation des systèmes

Il apparaît utile de compléter la liste précédente des mots-clés et concepts par les résumés descriptifs des modalités d'adaptation aux perturbations et autres contraintes. La figure 2 résume ces réponses sur les axes de repérage des structures et des finalités des systèmes (Alcaras et Lacroux 1999). Ces auteurs proposent en effet de définir les notions de rigidité, de flexibilité, d'élasticité et de plasticité d'un système par l'intermédiaire des aptitudes à modifier sa struc-

Les systèmes rigides ne s'adaptent pas aux contraintes externes. Ils ne déforment pas leur structure et ne modifient pas leur finalité... jusqu'à la ruptu-

grâce à leur capacité à modifier leur structure et à se refinaliser par rapport à un nouvel objectif. La plasticité se caractérise ainsi par la faculté de prendre forme et la capacité de changer de forme. L'évolution du monde vivant nous donne de multiples exemples de la plasticité des espèces en réponse à des modifications de contraintes de milieu.

La figure 3 illustre ces notions de façon schématique en complément de la figure 1 : en réponse à une perturbation, la trajectoire finalisée est maintenue (rigide), temporairement modifiée (élastique), ajustée (flexible) ou refinalisée (plastique). Le terme de vicariance apparaît également dans la littérature (Paillard 1976) pour qualifier la possibilité de trajectoires alternatives pour parvenir au même but.

Figure 3. Modalités des réponses dynamiques d'un système perturbé.

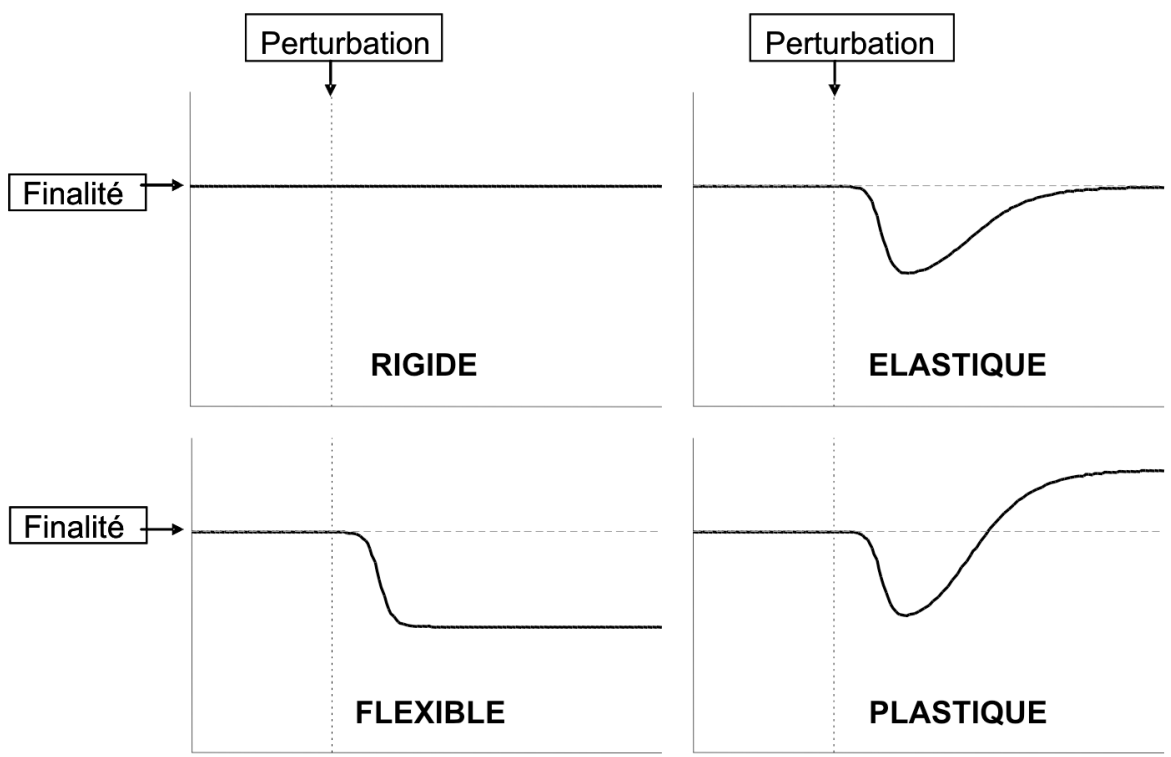




\section{4 / La qualification classique de l'adaptation des systèmes}

Il existe, dans tous les langages courants, donc dans les dictionnaires, des mots qui permettent de résumer les propriétés adaptatives des systèmes. Il s'agit en particulier des qualificatifs «robuste» et «rustique».

Un système robuste est un système fort, solide, ferme et résistant grâce à sa structure. On se rapproche ainsi du concept de rigidité. Cependant, dans le cas des organismes animaux, on peut dire que la robustesse tire son existence des régulations de son fonctionnement (cf. l'homéostase) et de la combinaison des propriétés d'élasticité, flexibilité... qui leur confèrent une capacité à s'adapter et à survivre. De façon analogue, on peut dire que les propriétés de plasticité assurent la pérennité des espèces malgré des modifications du milieu.

Un système rustique possède différentes propriétés en fonction des trois acceptions de la langue courante de ce qualificatif :

- un système rustique se définit par son caractère rural ou ancien,

- un système rustique se caractérise par sa rudesse et sa grossièreté,

- un système rustique est robuste, endurant, résistant aux perturbations et demandant peu de soins.

Dans le cas présent, c'est surtout la 3 ème acception qui nous concerne et qui attribue une certaine synonymie entre robustesse et rusticité. D'ailleurs, des textes de plus de 20 ans présentaient la rusticité en des termes qu'on appliquerait plutôt aujourd'hui à la robustesse, voire même à la résilience (Vallerand 1987). Généralement, le terme rustique fait référence à un système dont les exigences sont faibles : il est ainsi, par nature, peu affecté par des contraintes. Le terme robuste fait plutôt référence à un système capable de se maintenir face à des situations contraignantes : il est ainsi, grâce à ses réactions, peu affecté par des contraintes.

\section{2 / Approche systémique et régulations de la flexibilité, robustesse...}

\section{1 / Les principes}

Les systèmes considérés dans les sciences animales sont systématiquement structurés par différents niveaux d'organisation spatio-temporels. Les échelles spatiales sont, par exemple en remontant à travers les niveaux, la cellule, l'organe, l'organisme entier, le troupeau, le système de production, la région, la filière, etc. Les échelles temporelles correspondantes sont par exemple des minutes, heures, jours, semaines, mois, années, etc. Les grandes questions qui se posent à propos du comportement de ces systèmes, en particulier leur réponse adaptative, nécessitent de mieux connaître les régulations associées.

On peut dire a priori que tout l'arsenal des concepts de la systémique et des propriétés des systèmes et de leurs éléments contribue, d'une façon ou d'une autre, à leur robustesse qui apparaît dès lors comme un caractère émergent d'un système complexe. Par exemple, au niveau structurel, on trouve des fondements de propriétés adaptatives et de robustesse dans :

- l'existence de compartiments de stockage transitoire induisant soit un retard accompagné d'un amortissement, soit un relargage brutal de substance ou d'informations induisant une réaction rapide, soit une adaptation dynamique de fonctions d'offre et de demande,

- l'existence de structures générant des retards et capable d'en modifier la durée ou l'ordre, ce dernier influençant la capacité d'amortissement des perturbations,

- la possibilité d'avoir des flux déclenchés à partir d'une situation de seuils,

- l'existence de structures d'amont permettant au système d'anticiper l'organisation de sa réponse à une perturbation pré-identifiée,

- l'existence de procédés de tri et sélection au niveau des fonctions d'entrée ou de sortie du système. L'existence de phénomènes de vicariance internes permettant de mieux endiguer les effets d'une perturbation,

$$
\text { - etc. }
$$

Au niveau fonctionnel, les systèmes considérés possèdent en général, sous l'effet de leurs régulations, deux types de trajectoires associées : une trajectoire stratégique qui détermine l'évolution à long terme prédéfinie du système et, autour de cette trajectoire, des actions adaptatives d'ordre tactique qui permettent aux systèmes de rester dans le champ proche de la trajectoire stratégique malgré les perturbations de l'environnement. Ces aspects se retrouvent en ces termes au niveau des systèmes de production : dans ce cas, l'éleveur joue, par ses décisions stratégiques ou tactiques, le rôle de régulateur principal. Cette description initiale rejoint le concept de canalisation (Waddington 1957). Chez les êtres vivants, ces deux concepts se retrouvent à travers leur projet téléonomique (Monod 1970). Ainsi, les régulations d'homéorhèse (Bauman et Currie 1980), ou téléophorhèse (Chilliard 1986), sont finalisées par la pérennité de l'espèce d'appartenance. Elles concernent donc majoritairement les fonctions de reproduction. On y adjoint en général les fonctions de croissance qui correspondent à une phase de maturation avant d'atteindre la capacité à se reproduire. Le terme téléophorhèse (= transport/phorèse/ orienté vers une finalité/téléo/) est plus adapté à cette finalité biologique, et à la notion de projet téléonomique, que homéorhèse (écoulement/rhèse/constant/homéo/), mais ce dernier est plus utilisé au niveau de la littérature internationale. En synergie des régulations d'homéorhèse/téléophorhèse, les régulations d'homéostase permettent

Figure 4. L'articulation homéorhèse-homéostase dans l'espace des paramètres physiologiques, aspects dynamiques (Martin 2009).

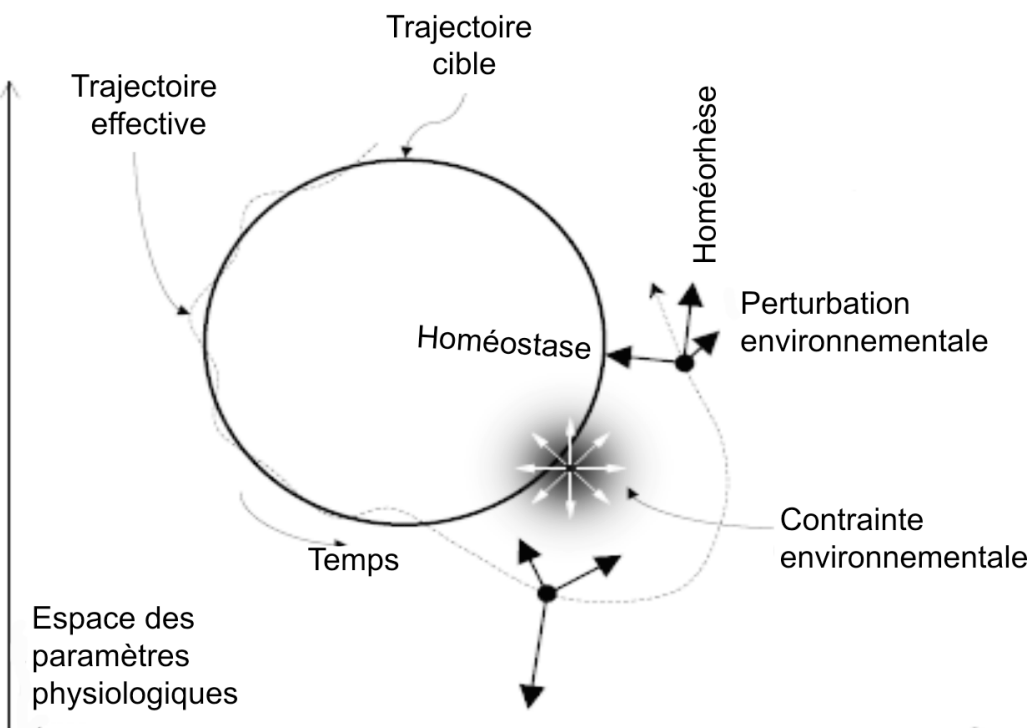


d'assurer la survie de l'organisme vivant considéré, malgré les nombreuses perturbations et contraintes environnantes, par la mise en œuvre de mécanismes de retour à un état d'équilibre (Sauvant et al 1983). Cette adaptation met en jeu des mécanismes physiologiques et comportementaux.

Vu sous un angle cinématique, un être vivant apparaît donc comme un point mobile dans un espace multidimensionnel, les dimensions correspondant aux variables d'état du système. Ce point mobile suit prioritairement une trajectoire génétiquement définie, que l'homéorhèse tend à préserver. Le terme chréode a été utilisé par Waddington (1957) pour définir cette trajectoire. Lorsque le système est perturbé, il s'éloigne de sa trajectoire et son retour à celle-ci, c'est-à-dire sa réponse adaptative, est permis par les forces d'homéostase et d'homéorhèse qui agissent aux abords de la trajectoire. La figure 4 résume la composition de ces deux forces pour définir la trajectoire effective (phénotypique) des êtres vivants.

Un autre point de vue, statique, de ce phénomène consiste à se placer orthogonalement à la trajectoire générée par l'homéorhèse/téléophorhèse, ce qui laisse alors apparaître la chréode comme un bassin d'attraction d'homéostase qui ramène le point mobile vers la trajectoire et définit ainsi la zone du domaine physiologique (figure 5). Lorsque la force perturbante fait sortir le système de ce bassin d'attraction, l'organisme entre dans un domaine de dysfonctionnement, souvent d'ordre pathologique. De multiples exemples permettent de retrouver ce type de réponse (adaptation aux températures, réponses aux repas ou à des nutriments non reconnus, réponses à une situation stressante ou aux maladies infectieuses...).

Ce schéma conceptuel peut se décliner dans les différentes disciplines. Par exemple en génétique, le modèle épistatique distingue les gènes qui gèrent l'expression du potentiel (cf. l'homéorhèse) de ceux qui gèrent la variabilité adaptative à l'environnement (cf. l'homéostase). Ces aspects sont précisés dans le texte de Bodin et al (2010) de ce même dossier.

Les êtres vivants constituent des systèmes complexes en raison de la multiplicité des niveaux d'organisation, des relations inter et intra-niveaux, du degré de complication de ces relations, etc. De ce fait, l'émergence des propriétés adaptatives, donc de la robustesse, n'est pas facile à comprendre. En particulier l'imbrication et les synergies entre des composantes d'élasticité, plasticité, rigidité... sont souvent quasi inextricables dans l'état de nos connaissances actuelles. Pourtant on sait que, par exemple, la rigidité observée à un niveau, ou sur un caractère, est en général obtenue grâce à une élasticité accrue réalisée à un autre niveau. On rejoint ainsi conceptuellement l'équilibre entre l'ordre et le désordre dans le vivant. Ceci montre au passage que les proprié- tés constitutives des systèmes, qui mettent en jeu celles de ses éléments et de leurs relations mutuelles, sont plus impliquées que les propriétés sommatives, qui ne mettent en jeu que les propriétés des éléments, dans la construction de la robustesse.

\section{2 / Evaluation et modélisation de la flexibilité, robustesse...}

Compte tenu de la complexité des systèmes et propriétés considérés (cf. ci-dessus), il est souvent utile de se placer successivement sous différents points de vue pour appréhender au mieux la robustesse d'un système objet d'étude, un organisme animal par exemple. A chaque point de vue considéré, pour évaluer la stabilité et la robustesse d'un système, il est nécessaire d'observer sa dynamique de réponse à la perturbation majeure relevant de ce point de vue. En outre, il est en général indispensable de considérer plusieurs variables caractérisant son fonctionnement, afin de tenter d'appréhender la mécanique du changement conduisant à un retour à l'équilibre ou à un nouvel équilibre. De plus, ces aspects soulèvent la question importante des indicateurs utilisables. En effet, il n'y a pas toujours d'indicateur pertinent d'un qualificatif à évaluer. La modélisation est un outil adapté pour explorer la dynamique de phénomènes impliquant des mécaniques sous-jacentes et des propriétés émergentes sous forme d'équilibre. L'enjeu d'un modèle capable d'intégrer la notion de robustesse est de pouvoir représenter

Figure 5. L'articulation homéorhèse-homéostase dans l'espace des paramètres physiologiques, vue statique (ou en coupe) et principe de la gouttière.

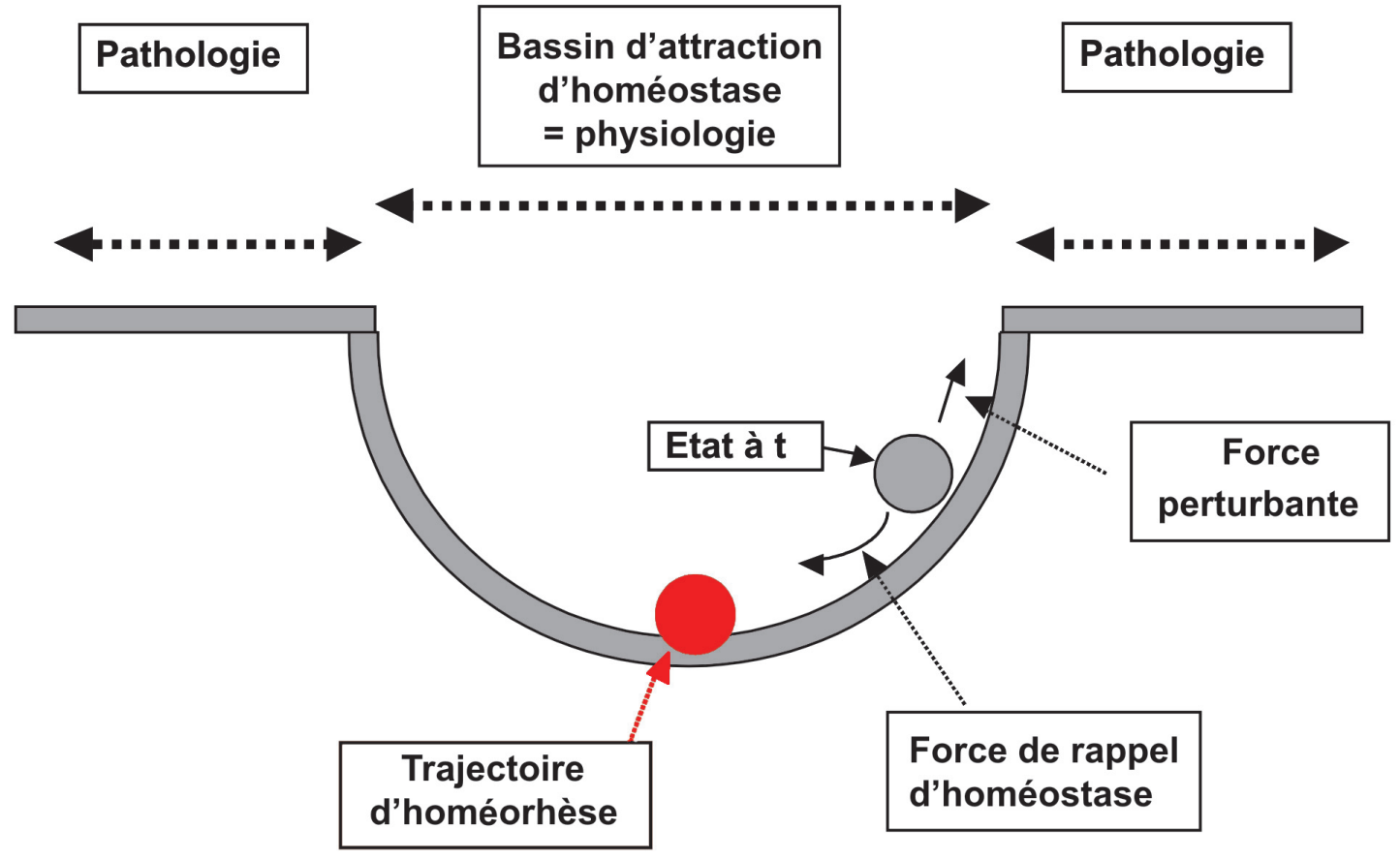


l'émergence d'une aptitude à résister aux changements externes à travers la capacité de moduler sa structure et/ou son fonctionnement interne. Cet objectif peut être partiellement atteint sur des exemples assez simples, par exemple dans le domaine de la digestion et de la nutrition des animaux. En revanche, il reste à construire un modèle mécaniste assez complet et spécifiquement capable d'explorer et de traduire la richesse des possibilités d'adaptation d'un organisme, ou d'un système de production, face aux perturbations auxquelles il est confronté.

\section{Conclusion}

Cet article introductif a permis de présenter les principales définitions et les principaux concepts rattachés à la notion de robustesse. Bien entendu, ces définitions peuvent varier sensiblement selon le secteur disciplinaire considéré, et nous nous sommes donc attachés à rester le plus générique possible. Il apparaît clairement que la robustesse fait partie de l'approche systémique et qu'elle constitue une émergence des systèmes complexes, dans le monde vivant en particulier. Elle doit donc être abordée en tant que telle, en évitant les raccourcis et mesures trop simplificateurs, donc en cherchant à mieux la comprendre sous différents points de vue et en respectant les différentes échelles d'organisation spatio-temporelles impliquées.

\section{Références}

Alcaras J.R., Lacroux F., 1999. Planifier, c'est s'adapter. Économie et Sociétés, série S.G., 26-27, 7-37.

Bauman D.E., Currie W.B., 1980. Partitioning of nutrients during pregnancy and lactation: a review of mechanisms involving homeostasis and homeorhesis. J. Dairy Sci., 63, 1514-1529.

Bodin L., Bolet G., Garcia M., Garreau H., Larzul C., David I., 2010. Robustesse et canalisation : vision de généticiens. In : Robustesse, rusticité, flexibilité, plasticité, résilience... les nouveaux critères de qualité des animaux et des systèmes d'élevage. Sauvant D., Perez J.M. (Eds). Dossier Inra Prod. Anim., 23, 11-22.
Chilliard Y., 1986. Variations quantitatives et métabolisme des lipides dans les tissus adipeux et le foie au cours du cycle gestation-lactation. 1 ère partie : chez la ratte. Reprod. Nutr. Dév., 26, 1057-1103.

Martin O., 2009. Modélisation systémique des performances des femelles de ruminants. Application au cas de la vache laitière. Thèse PhD, AgroParisTech, Paris, France, 343p.

Monod J., 1970. Le hasard et la nécessité. Essai sur la philosophie naturelle de la biologie moderne. Le Seuil, Paris, France, 219p.

Paillard J., 1976. Réflexions sur l'usage du concept de plasticité en neurobiologie. J. Psychol. Norm. Pathol., 1, 33-47.
Sauvant D., Soyeux Y., Chilliard Y., 1983. Réflexions sur l'étiopathogénie des maladies de la nutrition. Bull. Tech. CRZV Theix, 53, 117-121.

Vallerand, F., 1988, La rusticité, niveaux et méthodes d'approche en milieu réel. In : De la touffe au paysage. Hubert B., Girault N. (Eds), INRA-SAD, 85-101.

Waddington C.H., 1957. The strategy of the genes. A discussion of some aspects of theoretical biology. George Allen and Unwin, London, UK, 262p.

\section{Résumé}

La problématique globale de ce texte introductif concerne l'adaptation des systèmes aux perturbations. L'étude des modalités de cette adaptation engendre différents termes, notions et définitions. Ainsi, ces notions de rigidité, d'élasticité, de flexibilité et de plasticité... se retrouvent dans de nombreuses disciplines scientifiques, qu'il s'agisse de la physique, de la sociologie, de l'économie, de la biologie animale et des systèmes d'élevage. L'approche systémique permet de situer les facettes de l'adaptation des systèmes dans un cadre générique largement partagé et la modélisation de cette adaptation constitue un vrai défi aux chercheurs.

\section{Abstract}

Robustess, rusticity, flexibility, placticity... the quality new criteria of farm animals: systemic and biological definitions of the various concepts

The global topic of this introduction deals with the adaptation of systems to perturbations. The study of the modalities of this adaptation implicates various terms, notions and definitions. Thus, the notions of rigidity, elasticity, flexibility and plasticity... are known in numerous sciences: physics, sociology, economy, animal biology and farming systems. The systemic approach allows considering the various aspects of the adaptation of systems into a generic frame largely admitted and modelling this adaptation constitutes a current challenge to researchers.

SAUVANT D., MARTIN O., 2010. Robustesse, rusticité, flexibilité, plasticité... les nouveaux critères de qualité des animaux et des systèmes d'élevage : définitions systémique et biologique des différents concepts. In : Robustesse, rusticité, flexibilité, plasticité, résilience... les nouveaux critères de qualité des animaux et des systèmes d'élevage. Sauvant D., Perez J.M. (Eds). Dossier Inra Prod. Anim., 23, 5-10. 
